\title{
Trapping enhanced by noise in nonhyperbolic and hyperbolic chaotic scattering
}

\author{
Alexandre R. Nieto,, * Jesús M. Seoane, ${ }^{2}$ and Miguel A.F. Sanjuán²,3 \\ ${ }^{1}$ Nonlinear Dynamics, Chaos and Complex Systems Group, \\ Departamento de Física, Universidad Rey Juan Carlos, \\ Tulipán s/n, 28933 Móstoles, Madrid, Spain \\ ${ }^{2}$ Nonlinear Dynamics, Chaos and Complex Systems Group, \\ Departamento de Física, Universidad Rey Juan Carlos, \\ Tulipán s/n, 28933 Móstoles, Madrid, Spain \\ ${ }^{3}$ Department of Applied Informatics, Kaunas University of Technology, \\ Studentu 50-415, Kaunas LT-51368, Lithuania
}

(Dated: May 13, 2021)

\begin{abstract}
The noise-enhanced trapping is a surprising phenomenon that has already been studied in chaotic scattering problems where the noise affects the physical variables but not the parameters of the system. Following this research, in this work we provide strong numerical evidence to show that an additional mechanism that enhances the trapping arises when the noise influences the energy of the system. For this purpose, we have included a source of Gaussian white noise in the Hénon-Heiles system, which is a paradigmatic example of open Hamiltonian system. For a particular value of the noise intensity, some trajectories decrease their energy due to the stochastic fluctuations. This drop in energy allows the particles to spend very long transients in the scattering region, increasing their average escape times. This result, together with the previously studied mechanisms, points out the generality of the noise-enhanced trapping in chaotic scattering problems.
\end{abstract}

PACS numbers: 05.45.Ac, 05.45.Df,05.45.Pq

*alexandre.rodriguez@urjc.es 


\section{INTRODUCTION}

Chaotic scattering in open Hamiltonian systems is an important topic in nonlinear science due to its fundamental applications in classical [1-3] and quantum physics [4-6]. This phenomenon is also relevant in a wide variety of fields such as chemistry [7, 8], biology [9, 10] and even medicine [11]. Most of the work in chaotic scattering has been made by using purely conservative systems [12-15]. However, almost every system in nature is influenced by its surrounding environment. For that reason, recently some research has considered perturbations in the conservative systems as an attempt to model the coupling of the system with the environment. In particular, the effects of dissipation [16-18], periodic forcing [19, 20] and noise [21-24] have been considered. It has been shown that weak dissipation can convert the Kolmogorov-Arnold-Moser (KAM) islands into attractors, and that the periodic forcing can destroy the KAM structures at the same time that it decreases the unpredictability of the system [20].

Regarding the effects of noise, much work has been done in the recent years. Most of this research has focused on chaotic maps where an additive noise affects the physical variables of the systems. In this situation it has been shown that the noise can destroy the small scales of the KAM islands [25], leading to escapes that would be forbidden in the deterministic system [21, 22]. Nevertheless, the opposite effect can occur and trajectories that do not belong to the KAM islands can enter inside them and describe a transient regular motion [21, 24]. Furthermore, another interesting phenomenon has been reported: the noise-enhanced trapping [24]. Small noise intensities can play a constructive role by reducing the escape rate of the particles. The noise-enhanced trapping appears in both fully chaotic (hyperbolic) and mixed-phase-space (nonhyperbolic) systems, even if the mechanism that generates it is different in each case. In the fully chaotic case, the escape rate is reduced due to the blurring of the natural measure of the exits, and therefore allowing the trajectories to avoid their escape. In mixed-phase-space systems the trapping is enhanced by means of some trajectories that, even starting inside the chaotic sea, can enter the regions defined by the KAM islands in the noiseless case.

Regarding the effect of noise in continuous open Hamiltonian systems, we find less examples in the literature. In particular, one relevant work (see Ref. [23]) in which a threshold value of the noise intensity changes the decay law from algebraic to exponential in mixed-phase-space systems has been uncovered. The exponential decay remains ubiquitous for strong noise intensities [26]. In addition, some investigations of the effects of the noise into the fractal structures of the exit 
basins has been carried out, both in presence of Gaussian noise [23] and noisy periodic forcing [27]. However, as far as we know, the noise-enhanced trapping has not been the focus of attention in the context of the continuous Hamiltonian systems.

The main goal of our manuscript is to show that an additional mechanism to those described above takes place in open Hamiltonian systems. At the same time, we also confirm that the previously studied mechanism which occurs in mixed-phase-space maps is also relevant when the energy of the system is subject to fluctuations. Since most of chaotic maps are obtained as a Poincaré surface of section of continuous systems, the results are expected to be identical in both kind of systems. However, in open Hamiltonian systems the introduction of noise in the momentum or in the spatial coordinates indirectly affects the energy. This main difference allows a new and supplementary mechanism to occur. Surely, this mechanism is expected to play a certain role in chaotic maps where the noise affects the parameters of the system (e.g. multiplicative noise).

The mechanism that we have undercover appears in both hyperbolic and non-hyperbolic regimes and it is related to the possibility that stochastic fluctuations allow some trajectories, that we call unusual, to remain in the scattering region with low energy values during very long transients. The smaller the energy, the smaller the exit set of the system and, in consequence, higher the escape times.Moreover, for low energy values the phase space of many systems is occupied by extensive KAM tori, so the noise that enhances the trapping allows the trajectories to enter in KAM regions of the deterministic system that exist for different values of the energy. In this sense, the new mechanism reported in this manuscript can be understood as a catalyst of the previously investigated one.

To study this issue, we have chosen the Hénon-Heiles system [28], which represents a paradigmatic example of chaotic scattering that exhibits a transition between hyperbolic and nonhyperbolic dynamics. This characteristic is interesting because we can analyze the noise-enhanced trapping in fully chaotic and mixed-phase-space cases using the same model.

The structure of this paper is as follows. In Sec. III, we describe our model, the Hénon-Heiles system with additive uncorrelated Gaussian noise. In Sec. [II and Sec. IV we show strong numerical evidence of noise-enhance trapping in the fully chaotic and mixed-phase-space regimes of the system, respectively. In the latter, we also show the gradual reduction of the stickiness of the KAM islands under the effect of very weak noise. The explanation about the mechanism leading to the trapping is carried out in Sec. $\mathrm{V}$. Finally, in Sec. VI, we present the main conclusions of this manuscript. 


\section{MODEL DESCRIPTION}

The model that we have used to study the noise-enhanced trapping in continuous open Hamiltonian systems is the Hénon-Heiles. This Hamiltonian system appeared in the literature for the first time in 1964 as a model of a particle moving in a galactic potential. The Hamiltonian is given by the sum of the kinetic energy and a nonlinear axisymmetric potential [see Fig. 11(a)]:

$$
\mathcal{H}=\frac{1}{2}\left(p^{2}+q^{2}\right)+\frac{1}{2}\left(x^{2}+y^{2}\right)+x^{2} y-\frac{1}{3} y^{3},
$$

where $x$ and $y$ are the coordinates, and $\dot{x}=p$ and $\dot{y}=q$ denote the two components of the generalized momentum.

(a)

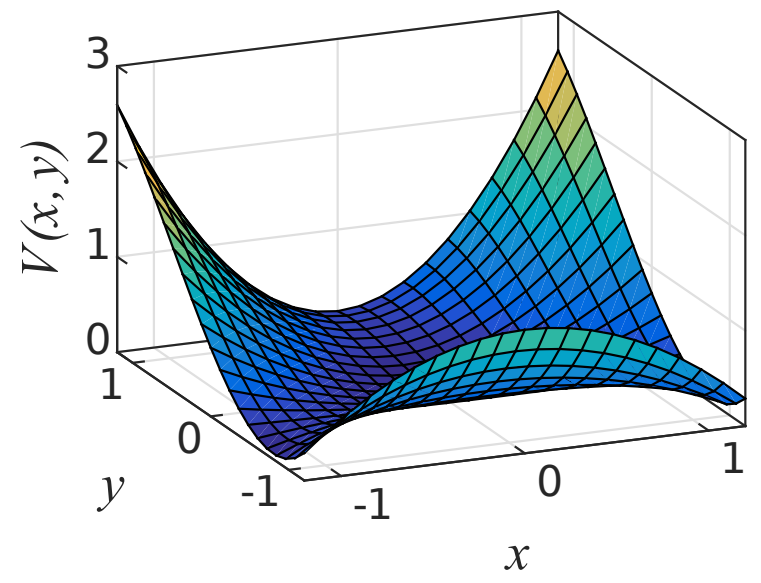

(b)

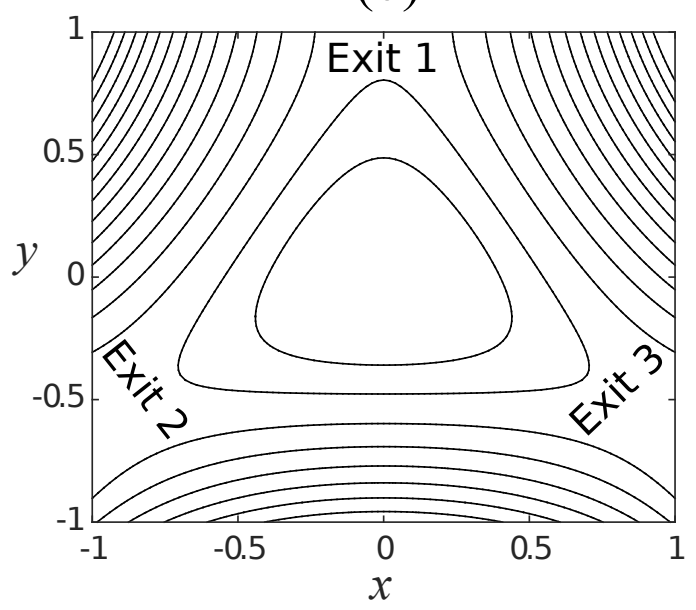

FIG. 1. (a) Potential $V(x, y)$ of the the Hénon-Heiles system. (b) Isopotential curves for different values of the energy, under and over the threshold value $E_{e}=1 / 6$. For energy values $E>E_{e}$ three different exits are visible: exit $1(y \rightarrow \infty)$, exit $2(x, y \rightarrow-\infty)$, and exit $3(x \rightarrow \infty, y \rightarrow-\infty)$.

The system has been extensively studied due to the wealth of dynamical behaviors that exhibits for different values of the energy [29-32]. Particularly, the system exhibits two energy thresholds that separate very different regimes. The first threshold occurs when the energy is $E_{e}=1 / 6$.

For energy values below $E_{e}$ the isopotential curves are close, while for energy values over $E_{e}$ three exits separated by an angle of $2 \pi / 3$ appear. Then the particles can escape from the scattering region to infinity and the system becomes an open Hamiltonian system. To visualize this fact, we depict in Fig. 1(b) the isopotential curves for different values of the energy, under and over $E_{e}$. For energy values slightly higher than $E_{e}$ the mixed-phase-space is occupied by enormous 
KAM islands that make the dynamical behavior of the system nonhyperbolic. The nonhyperbolic character manifests, among other ways, through an algebraic decay law in the survival probability of the particles. If we increase the energy more and more, the size of the KAM islands decreases irregularly until $E_{t} \simeq 0.23$ [33], which is the second important threshold. Over $E_{t}$ all the KAM tori have been destroyed and the hyperbolic regime starts. This regime is characterized by an exponential decay law and a fully chaotic phase space.

For the purposes of this research we include in the Hénon-Heiles system a source of additive uncorrelated Gaussian noise. Under this consideration the equations of motion read [26]:

$$
\begin{aligned}
& \dot{p}=-x-2 x y+\sqrt{2 \xi} \eta_{x}(t) \\
& \dot{q}=-y-x^{2}+y^{2}+\sqrt{2 \xi} \eta_{y}(t),
\end{aligned}
$$

where $\xi$ is the intensity of the noise and $\eta_{x}(t), \eta_{y}(t)$ are Gaussian white noise processes with mean $\mu=0$ and variance $\sigma^{2}=2 \xi$.

To solve numerically this system of stochastic differential equations we have used the stochastic second-order Heun method [34], as was previously used in Refs. [23, 26]. To ensure the effectiveness of the method we have tested the stability of the solutions and the convergence of the main magnitudes of this research (average escape time and energies). Since the Heun method is not a symplectic algorithm and we have worked with a conservative system, we have included a negligible amount of dissipation in order to avoid stability problems in the integration algorithm. We have also compared the results by using the numerical schemes of the Euler-Maruyama method and the stochastic fourth-order Runge-Kutta method [34]. All the three methods have provided similar results with a good precision. However, the Heun method has been the one that offers the best balance between precision and computational effort.

\section{NOISE-ENHANCED TRAPPING IN THE FULLY CHAOTIC REGIME}

In this section, we study the phenomenon of noise-enhanced trapping in the fully chaotic Hénon-Heiles system. As we commented in the introduction, the last significant KAM torus is destroyed at the energy level $E_{t}=0.23$. However, it has been shown that the particles that belong to KAM islands are completely residual for energies above $E=0.21$ [33] and the phase space can be considered fully chaotic. For that reason, we have decided to consider in this section energies $E>0.21$ instead of $E>0.23$. The results of the next section will show that this division of 
energies is natural when studying the effect of noise in this system.

The main magnitude that we have studied to characterize the dynamical behavior is the average escape time of the trajectories, T. Because the system is 4-dimensional and we have to deal with 3 free coordinates, there exist many methods to launch trajectories in phase space in order to obtain $T$. Some researchers fix the physical coordinates as $(x, y)=(0,0)$ and then vary the angle of the launching. Other authors prefer to fix the angle and vary the physical coordinates over a line segment in the physical space. In order to consider a more general range of initial conditions in phase space, here we have used the tangential shooting method [15]. Using this method, the velocity vector is defined in such a way that is tangent to the circle centered at the origin and passing through the point $\left(x_{0}, y_{0}\right)$. This consideration implies that when we vary $\left(x_{0}, y_{0}\right)$ we are also varying $\left(p_{0}, q_{0}\right)$, so we are exploring initial conditions in all the phase space. To choose a suitable method to define the initial conditions is important, especially in the mixed-phase-spaceregime, because it is possible to miss some parts of the dynamical behavior (trapped trajectories for example) by choosing specific sets of initial conditions.

Now, we are going to describe the relevant results of this section. We have considered a wide range of noise intensities $\xi \in\left[10^{-10}, 10^{-1}\right]$ and computed the average escape times of the particles for energies $E=0.23$ and $E=0.25$. The results are shown in Fig. 2, where we can observe a maximum in the average escape time, showing that for specific values of the noise, $\xi_{t}$, the trapping is enhanced. For very weak noise intensities the escape times remain unaltered, while for very strong noises the escape time decreases abruptly.

To show these results in a more general manner, we have computed the average escape times for 40000 combinations of energies $E \in[0.21,0.25]$ and noise intensities $\xi \in\left[10^{-7}, 10^{-2}\right]$. To visualize the result we have used in Fig. 3, a color-coded map in which hot colors indicate high average escape times. As we could also observe in Fig. 2, this color-coded map shows that the value of $\xi_{t}$ increases with the energy, at the same time that the intensity of the trapping is reduced. This implies that if the energy is increased to values deep in the fully chaotic regime, the effect of the trapping in the average escape times might be negligible. In this situation the traditional mechanism of trapping [24], based on a reduction of the escape rate due to the blurring of the natural measure of the system, is expected to dominate the trapping phenomenon of the particles in the scattering region.

In chaotic maps with leaks, the noise enhances the trapping by blurring the measure of the leak $I$. The noise can move trajectories out of $I$, so they do not escape in the next iteration. In the case 

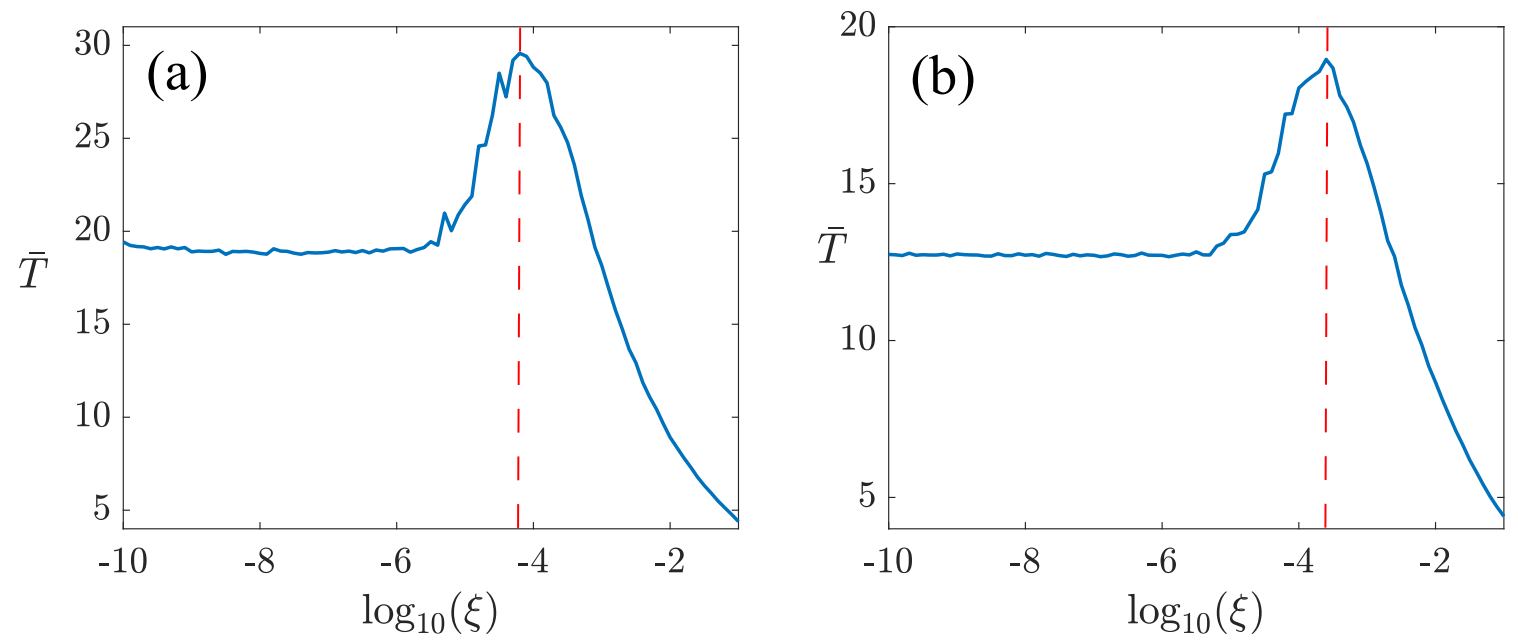

FIG. 2. Evolution of the average escape time with increasing noise for (a) $E=0.23$ and (b) $E=0.25$. The red dashed lines are located at the maximum of the average escape time. In order to compute this figure, we have used 200 values of $\xi \in\left[10^{-10}, 10^{-1}\right]$. For every noise intensity, we have launched 250000 initial conditions and calculated the average escape time.

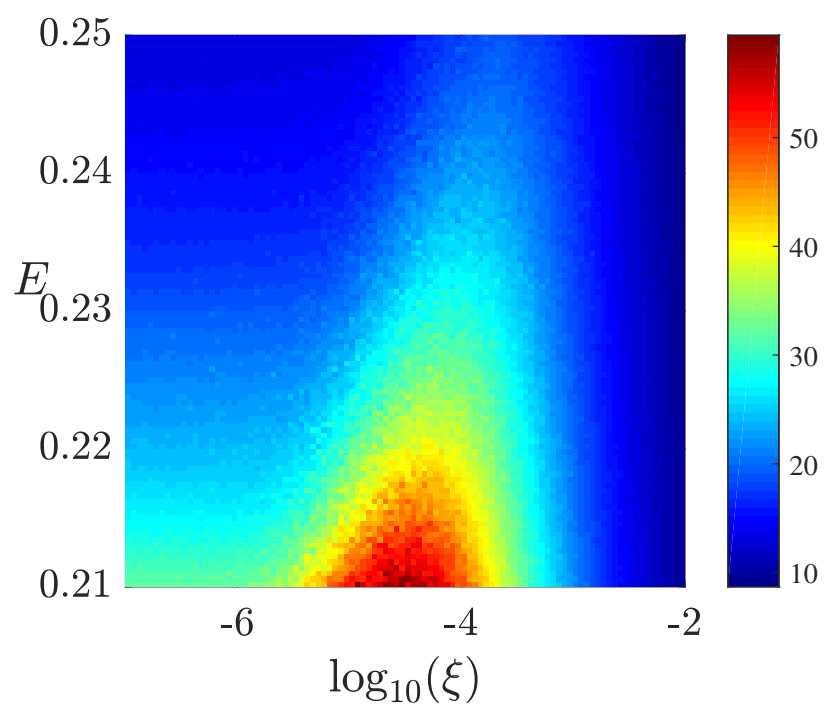

FIG. 3. Color-code map showing the average escape times for several values of the energy and the noise intensity. The hot colors indicate high values of the escape times. We have used $200 \times 200$ equally spaced values of the parameters. For every combination of energy and noise we have computed 50000 trajectories in order to calculate the average escape time. The figure clearly shows a noise intensity that allows the particles to describe unusual long transients in the scattering region. 
of continuous open Hamiltonian systems, the exit set is defined by the highly unstable periodic orbits called Lyapunov orbits [35]. Once a particle cross a Lyapunov orbit with the velocity vector pointing out, low noise intensities cannot make the particle to climb the hill and return to the scattering region. In particular, for energies $E \in[0.23,0.25]$ our simulations have established that only noise intensities $\xi>0.1$ allow some trajectories to return to the scattering region after crossing slightly a Lyapunov orbit.

\section{NOISE-ENHANCED TRAPPING IN THE MIXED-PHASE-SPACE REGIME}

In this section we analyze the noise-enhanced trapping in the mixed-phase-space regime of the system. We have started our computations in $E=0.18$, even if the exits are open over $E_{e}=1 / 6$. We have decided this to avoid the immense computational effort that is necessary when dealing with the convergence of the trajectories for energy values very close to $E_{e}$.

In Fig. 4 we show the evolution of the average escape time in function of the noise intensity for four energy values within the nonhyperbolic regime. Here, we can observe local maxima associated with the trapping for noise intensities between $10^{-4}$ and $10^{-6}$. The main qualitative difference between this figure and Fig. 2 is that an initial decrease in $T$ appears for energies 0.18 , 0.19 and 0.20 . As we will show, this decrease is directly related to the reduction of the stickiness of the KAM islands due to he effects of noise. In the case $E=0.21$ no decrease is observed due to the small size and influence of the KAM islands.

Strictly speaking, KAM islands do not exist in presence of noise. However, their ghosts manifest through their stickiness which can retain the particles during long transients. As the noise intensity is increased the stickiness is reduced, so the higher the noise the higher the probability of escaping from a KAM region in a certain time. A good way to visualize this reduction in the stickiness is through the exit basins [36]. In the context of continuous open Hamiltonian systems, we define an exit basin as the set of initial conditions that after a finite time will escape through a certain exit. In order to visualize them in a figure, we establish a different color to the initial conditions depending on the exit through which they will escape. We use another color, usually black or white, to represent the trajectories that belong to a KAM torus and do not escape. In Fig. 5 we represent (a) the exit basins in the physical space $(x, y)$ for $E=0.18$, and (b) a zoom-in in the exit basins, centered in one of the main three KAM islands of the deterministic system.

The effect of a source of noise weaker than the value that enhances the trapping is to allow 

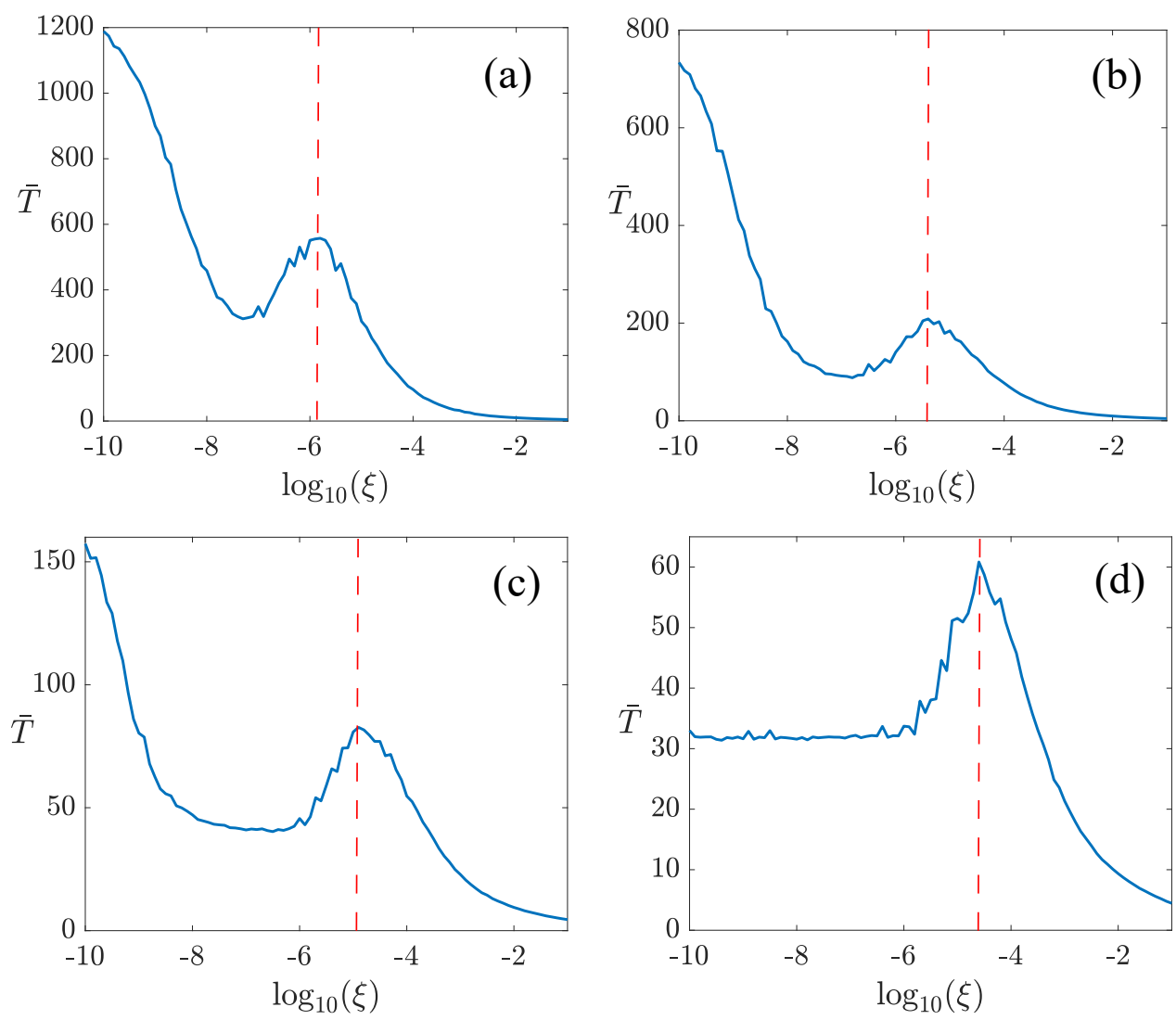

FIG. 4. Evolution of the average escape time with increasing noise for (a) $E=0.18$, (b) $E=0.19$, (c) $E=0.20$ and (d) $E=0.21$. The red dashed lines are located at the relative maxima of the average escape time. In order to compute this figure we have used 200 values of $\xi \in\left[10^{-10}, 10^{-1}\right]$. For every noise intensity we have launched 250000 initial conditions and calculated the average escape time. The maximum integration time that we have considered is $t_{\max }=100000$. In (a-c) panels a decrease in $T$ due to the progressive destruction of the stickiness of the KAM islands is observed. The average escape time of the deterministic system are $T_{a}=1314, T_{b}=792, T_{c}=161$, and $T_{d}=33$.

particles inside the KAM islands to leave the region and escape, so a blurring in the KAM regions in the exit basins is expected. However, the intensity of the blurring that we observe depends on the maximum integration time that we consider. In Fig. 6 we show the same zoom-in of Fig. 5(b), for different noise intensities and different maximum integration times. By observing the pictures from left to right (increasing $t_{\max }$ ) it is clear that the KAM islands are gradually blurred. In fact, we have tested that for $t_{\max }=50000$ there are no trapped particles in phase space. On the other hand, by observing the pictures from top to bottom (increasing the noise) we detect a faster blurring for higher noise. The higher the noise, the greater the reduction of the stickiness of the 
(a)

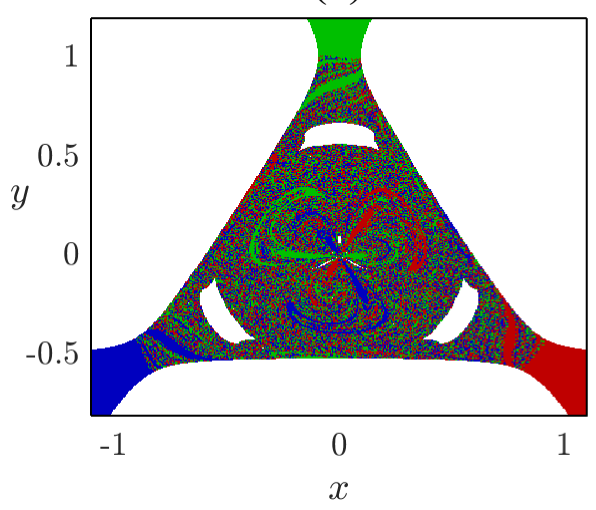

(b)

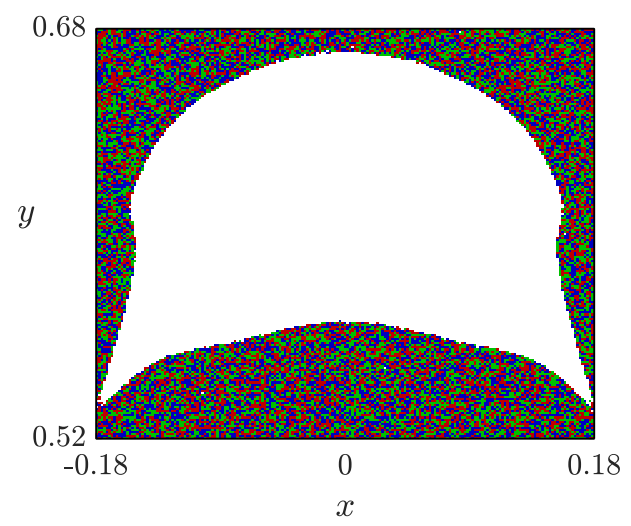

FIG. 5. (a) Exit basins in the physical space $(x, y)$ for the Hénon-Heiles system with energy $E=0.18$. The colors green, blue and red correspond to initial conditions escaping through exists 1,2 and 3 , respectively. The white color refers to particles that belong to a KAM torus and never escape. (b) Zoom-in on the KAM island which is close to the exit 1 . To compute this figure we have used (a) $400 \times 400$ and (b) $200 \times 200$ initial conditions.

KAM islands, which generates the decreasing in the average escape times that we have observed in Fig. 4

Even if Fig. 6 is clear enough for its purpose, it is worth noting that the exit basins have no meaning when working with noisy systems. Due to the effect of noise in the chaotic behavior, the same initial condition can escape through a different exit in different launchings. This implies that the color that appear in the exit basins can be different in different simulations. Being rigorous, the appropriate representation of the asymptotic behavior is by using probabilities. In particular, we can generate figures similar as Fig. 6 many times (say 100) and calculate the probability that a particle remains in the KAM island after the maximum integration time. This is exactly what we show in Fig. 7, where the yellow color means probability 1, while dark blue means probability 0. Intermediate colors imply intermediate probabilities. From this figure, we can state similar conclusions as in Fig. 6. For $\xi=10^{-9}$ the boundary of the KAM islands starts to have the possibility to escape when we increase the maximum integration time. In the case of $\xi=5 \times 10^{-8}$ almost all initial conditions have an option to escape for $t_{\max }=5000$, while for $t_{\max }=50000$ the probability to remain in the scattering region is almost zero for every initial condition. This figure serves just as another evidence of the gradual reduction of the stickiness for weak noise.

The fact that the stickiness of the KAM islands is reduced with increasing noise does not imply 
(a)

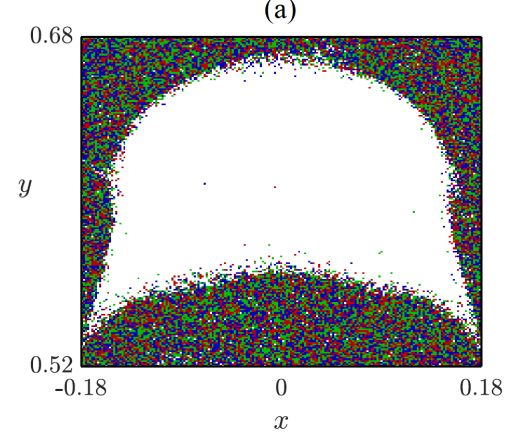

(d)

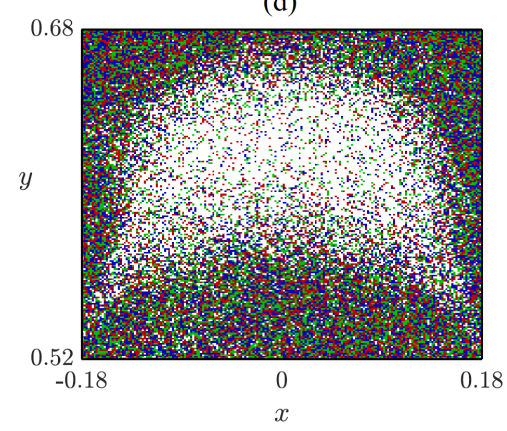

(b)

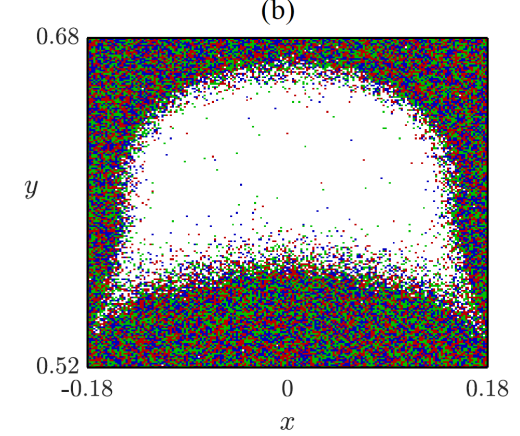

(e)

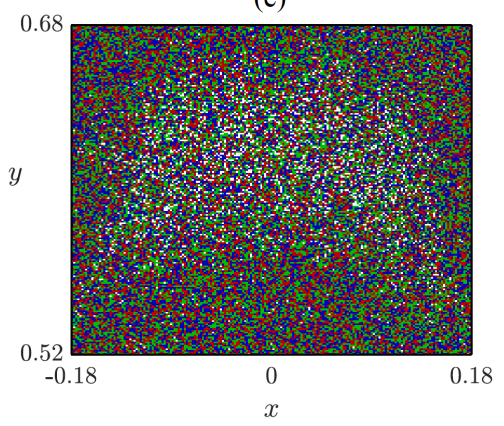

(c)

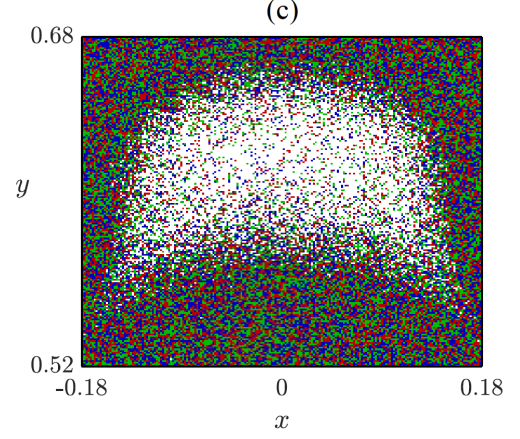

(f)

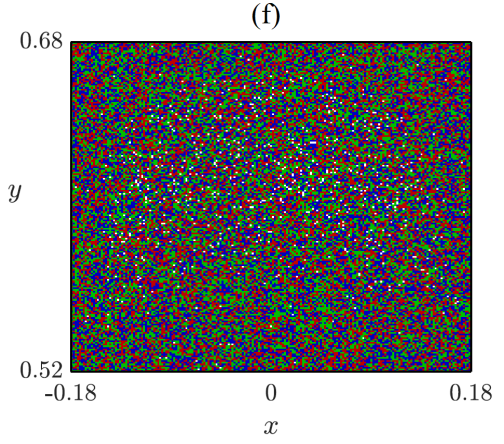

FIG. 6. Zoom-in of the exit basins of the Hénon-Heiles system with energy $E=0.18$. The intensities of the noise are $\xi_{a}=\xi_{b}=\xi_{c}=10^{-9}$ and $\xi_{d}=\xi_{e}=\xi_{f}=5 \times 10^{-8}$. The maximum integration time is $t_{a}=t_{d}=1000, t_{b}=t_{e}=5000$ and $t_{c}=t_{f}=20000$. The structure of the KAM regions is progressively blurred when increasing both the noise intensity and the maximum integration time. We have checked in all the cases that there are no trapped particles after $t_{\max }=50000$.

that they do not affect the escape dynamics in the noise-enhanced trapping. On the contrary, as it has been shown in previous works [24], due to the effects of noise, particles that describe escaping orbits in the deterministic system can jump into the KAM islands describing long transients. Hence, the initial decrease on the average escape time is explained by the reduction of the stickiness, but the relative maximum (i.e. the noise-enhanced trapping) is strongly related to the KAM structures. In continuous open Hamiltonian systems, changes in the physical variables due to the noise imply changes in the energy, so the particles are able to enter in the structure of the existing KAM islands for different values of the energy. However, this does not happen for every noise intensity, but only for the intensities that suit the characteristics of the fine structure of the KAM islands.

To illustrate the above reasoning, we have computed the escape time distributions for a very weak noise intensity $\xi=10^{-10}$ and the noise intensity that enhances the trapping for $E=0.18$ 
(a)

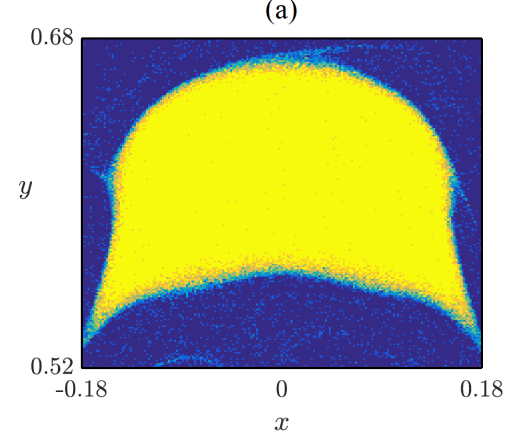

(d)

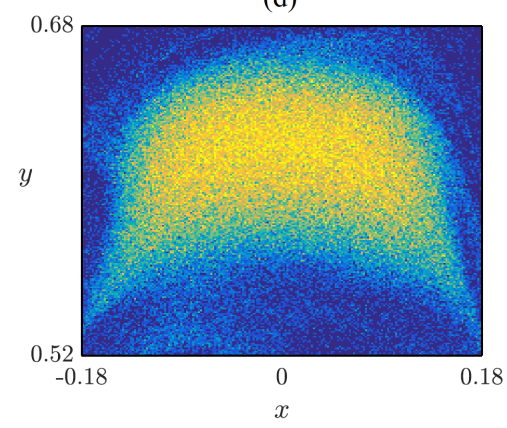

(b)

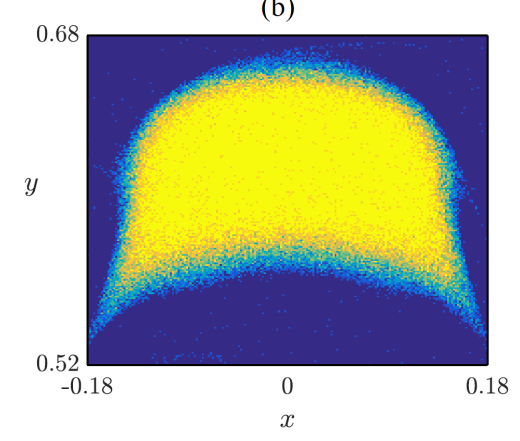

(e)

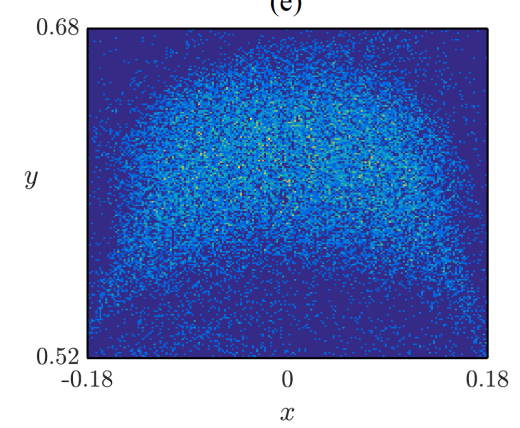

(c)

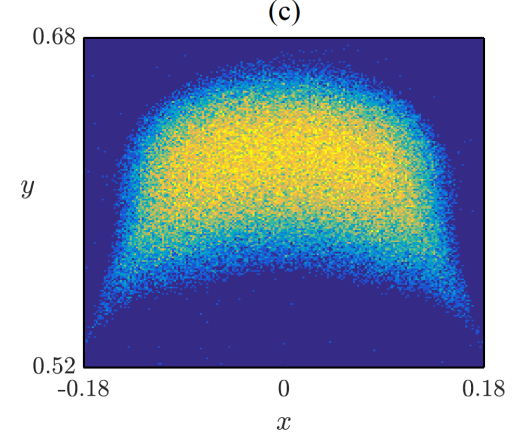

(f)

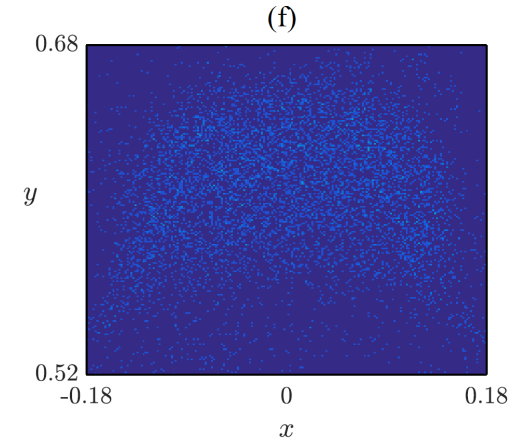

FIG. 7. Color-code showing the probability that an initial condition close to the KAM regions in the physical space for $E=0.18$ remains in phase space once the maximum integration time is reached. Yellow color means probability 1 , dark blue means probability 0 , and the other colors refer to intermediate probabilities. The intensity of the noise is $\xi_{a}=\xi_{b}=\xi_{c}=10^{-9}$ and $\xi_{d}=\xi_{e}=\xi_{f}=5 \times 10^{-8}$. The maximum integration time is $t_{a}=t_{d}=1000, t_{b}=t_{e}=5000$ and $t_{c}=t_{f}=20000$. To generate this figure we have used 100 exit basins and computed the probability for every initial condition. It is clear that the probability decreases with both the maximum integration time and the noise.

$\left(\xi_{t}=1.26 \times 10^{-6}\right)$. The result can be observed in Fig. 8. For the weakly noisy case, the particles that spend long transients in the scattering region are located in the vicinity of the KAM regions of the deterministic system (see Fig. 5 for comparison). This means that the particles starting far away from KAM tori cannot enter inside them, so they will escape in similar times than in the deterministic case. The result depicted for $\xi_{t}$ is drastically different. The KAM regions appear blurred and an important part of the basin seems smeared by particles with high escape times. The structure of the KAM region for $E=0.18$ is contained in the high escape time region, but they are not identical. In fact, the extensive yellow region consist of different KAM regions appearing for all the values of the energy. This happens because the trajectories can not only enter the KAM tori for $E=0.18$, but all the ones that appear for different energy values. 

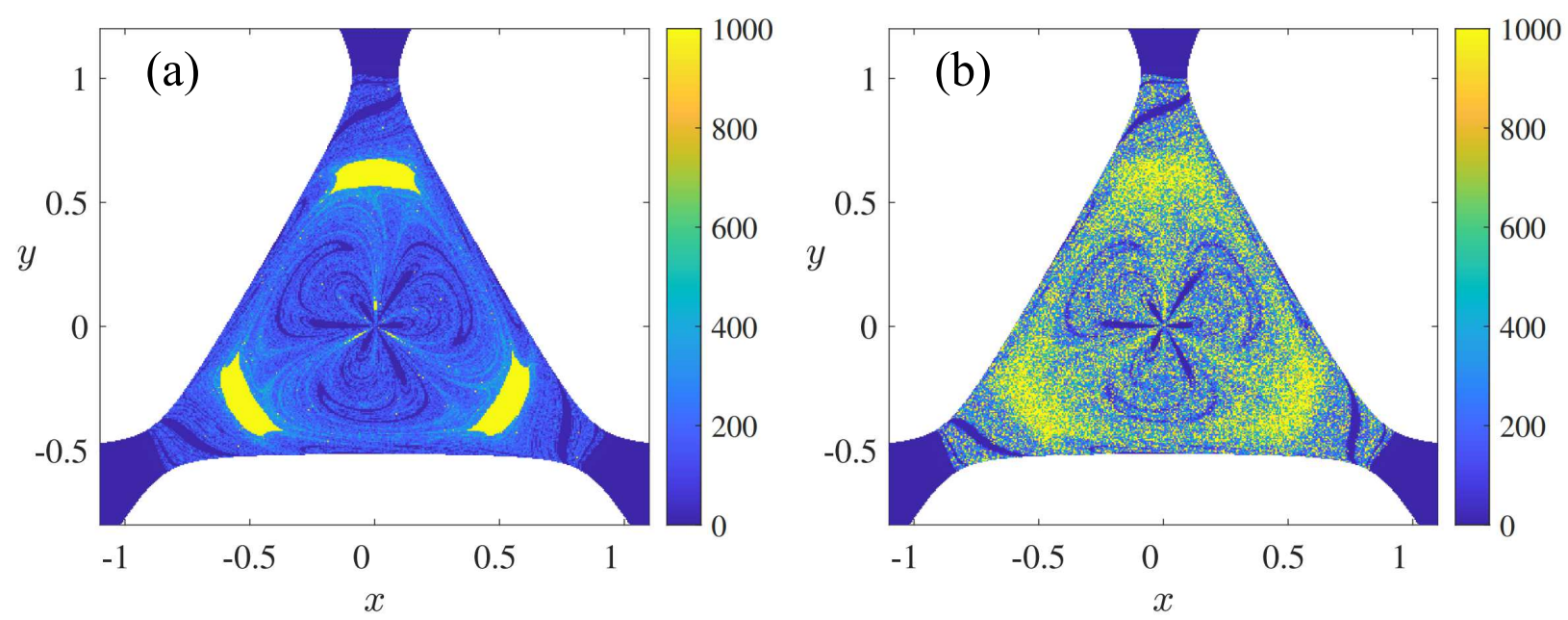

FIG. 8. Average escape time distribution for the Hénon-Heiles system with energy $E=0.18$ and noise intensities (a) $\xi=10^{-10}$ and (b) $\xi_{t}=1.26 \times 10^{-6}$. To generate these figures we have computed in the grid the average escape time of every initial condition 50 times, and calculated the average. As it can be seen in the color bar, hot colors indicate high average escape times, while cold colors refer to low escape times.

The entrance of escaping trajectories inside the KAM tori due to the noise is a well-known result. However, the possibility to enter in KAM islands existing for lower energy values is a new phenomenon that is possible because of the drop in energy. A particular noise intensity allows the particles to enter in the structure of the KAM islands, and the fluctuations in the energy catalyze this process. This is the main mechanism that explains the noise-enhanced trapping in open Hamiltonian systems, and we will discuss it in detail in the next section.

This relevant phenomenon can be illustrated by a simple representation in the physical space. In Fig. 9 (a) we show an escaping trajectory in the deterministic system with energy $E=0.18$, escaping after a short time $T=185$. In the rest of the panels we represent a trajectory starting in the same initial condition but affected by the noise-enhanced trapping. In each panel, we show a different time period of its evolution in order to make clear that the particle does not move in a single KAM torus but in many different ones, jumping from one to another. Panel (b) shows the whole evolution of the trajectory inside different KAM tori $(t \in[1000,10000])$. Panels (c), (d) and (e) illustrate the bounded motion with different energy during the time intervals $t \in[1000,1100]$, $t \in[5000,5100]$, and $t \in[9000,9100]$ respectively. Finally, panel (f) displays the escape of the trajectory after jumping out from the KAM tori. We have carried out numerical simulations showing that this phenomenon takes place also in the hyperbolic regime of the system. 

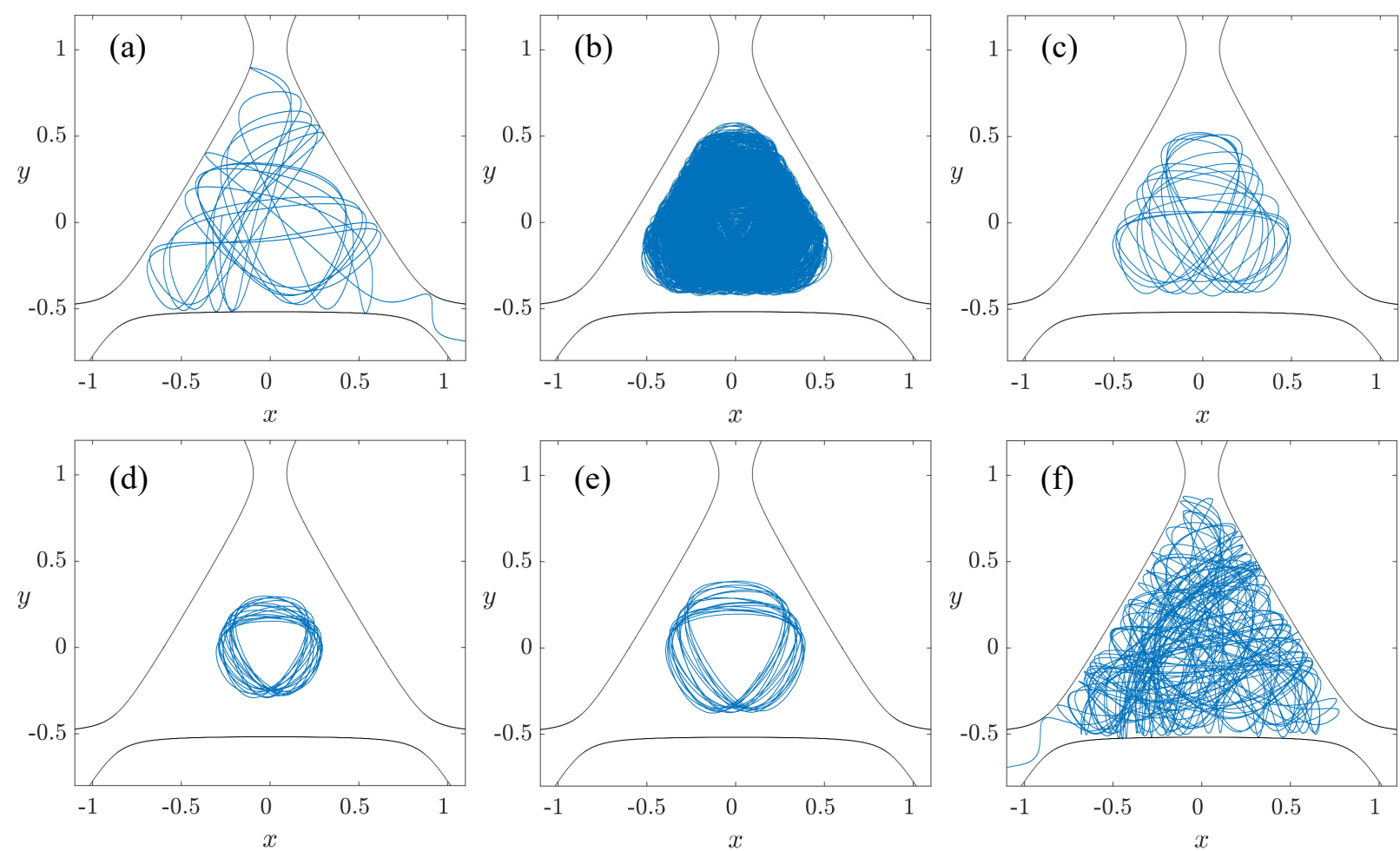

FIG. 9. Evolution of two trajectories starting in the same initial condition in the (a) deterministic and (b-f) noisy Hénon-Heiles system with energy $E=0.18$. The noise intensity is the one that enhances the trapping, $\xi_{t}=1.26 \times 10^{-6}$. Each panel shows a different time period of the evolution of the same trajectory. In particular, the time periods are (b) $t \in[1000,10000]$, (c) $t \in[1000,1100]$, (d) $t \in[5000,5100]$, (e) $t \in[9000,9100]$, and (f) $t \in[10000,10998]$. The escape time of the deterministic case is $T=185$.

To finalize the results in the mixed-phase-space regime, in Fig. 10 we use again a color-coded map to show a general portrait of the noise-enhanced trapping. The result is quite similar to that of Fig. 3, In fact, Fig. 3 is a natural continuation of this figure for higher energies.

\section{THE MECHANISM OF NOISE-ENHANCED TRAPPING}

In the previous sections, we have shown that a noise-enhanced trapping phenomenon appears in both the fully chaotic and mixed-phase-space regimes. Furthermore, we have provided some numerical evidence to show that regular trajectories of the deterministic system can enter in regions under the influence of KAM tori due to the effects of noise. These particles, that could escape in short times in the absence of noise, describe very long transients and generate a relative maximum in the evolution of the average escape time. In systems where the energy (or another relevant 


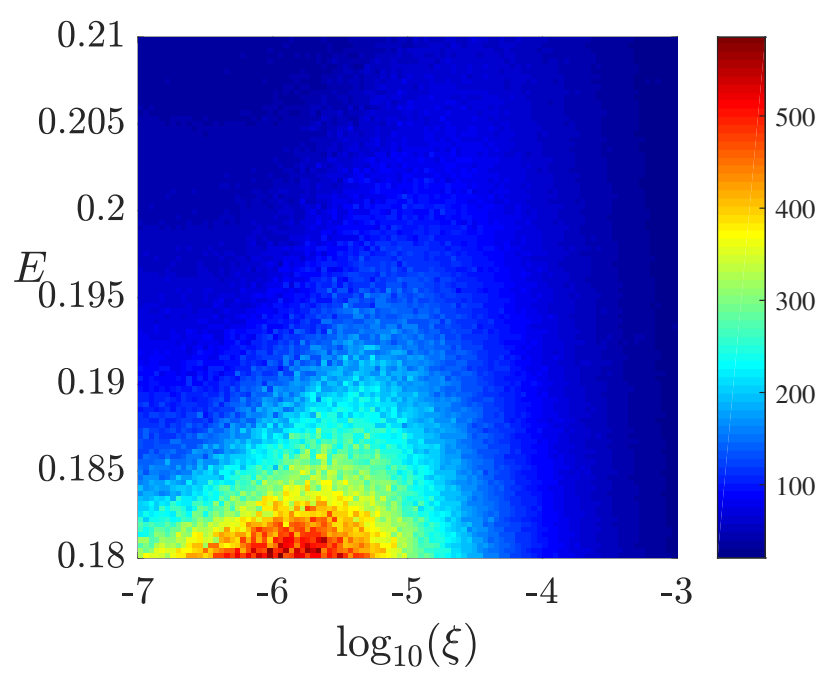

FIG. 10. Color-code map showing the average escape times for several values of the energy and the noise intensity. The hot colors indicate high values of the escape times. We have used $200 \times 200$ equally spaced values of the parameters. For every combination of energy and noise we have computed 50000 trajectories in order to calculate the average escape time. The figure clearly shows that the value of the noise that enhances the trapping decreases with decreasing energy.

parameter) is not influenced by the noise, the mechanism leading to the trapping is well known, as we have discussed in the Introduction of this manuscript. However, in the context of open Hamiltonian systems, a deep analysis on the energy fluctuations is necessary to understand the trapping phenomenon. We have tested the following results with several energies in the range $E \in[0.18,0.25]$. Nevertheless, for illustrative purposes, we will show here the simulations of the noisy system with initial energy $E=0.23$. In order to analyze what is happening with the trapping particles, we represent in Fig. 11 a histogram showing the escape time distribution in short times, in (a) the deterministic case and in (b) the noisy case with $\xi_{t}$. Even though the results are quite similar until $T=100$, the maximum escape time in the deterministic case is $T=207$, while in the trapping is $T=3887$ [see inset in Fig. 111(b)]. Hence, we can conclude that some unusual trajectories remain in the scattering region during very long transient and enhance the trapping.

We have analyzed these unusual trajectories and found the main characteristic that differentiate them from the usual trajectories that escape in short times: their average energy. In the deterministic system the energy is fixed by the Hamiltonian and only a negligible decrease (on the order $10^{-13}$ using our numerical scheme) appears after long integration times due to the numerical 
(a)

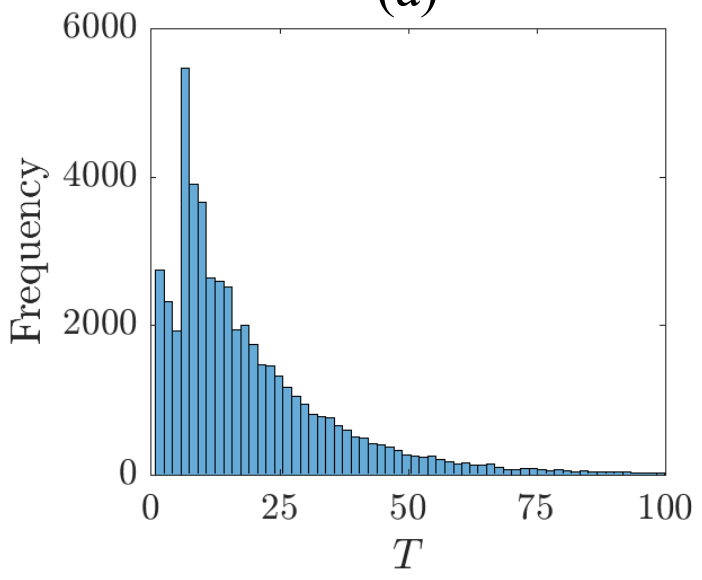

(b)

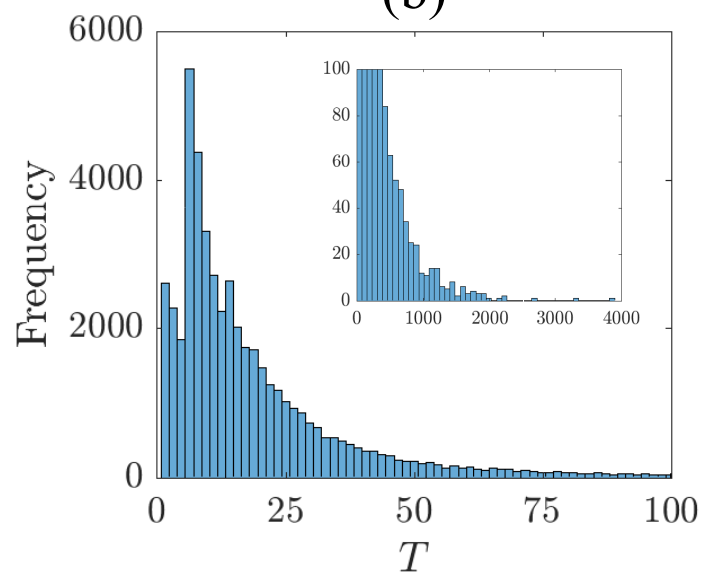

FIG. 11. Histogram showing the escape time distribution for (a) $\xi=0$ and (b) $\xi_{t}=6.31 \times 10^{-5}$. The initial energy of the system is $E=0.23$. In the deterministic case the maximum escape time is $T=207$, while for $\xi_{t}$ a small number of trajectories survive even until $T=3887$, as shown in the inset. To generate these histograms we have launched 50000 initial conditions in phase space and calculated the average escape time for all of them. The average escape time is $T=19.24$ for $\xi=0$ and $T=29.55$ for $\xi_{t}=6.31 \times 10^{-5}$, so the trapping is enhanced.

noise. However, in the presence of noise the energy is not preserved anymore. Because of the noise, particles can move with an average energy higher or lower than the initial one, $E_{0}$. Even so, in the white noise process the mean is zero, so it makes sense to think that a similar number of particles have an average energy $\bar{E}=E_{0}+\Delta E$ and $\bar{E}=E_{0}-\Delta E$. This reasoning is valid for low noise intensities. In Fig. 12(a) we use a histogram to show the average energy distribution in the presence of a weak noise intensity $\xi=10^{-6}$. We can clearly observe a Gaussian distribution centered on $E_{0}=0.23$ and energies ranging from $E=0.22$ to $E=0.24$. This means that the weak noise cannot drastically change the average energy, leading to values that could influence the average escape times. What happens is simply that some trajectories have a slightly higher or lower energy, but on average they escape with the same times as the deterministic system. As a visual example, the evolution of the average energy for three particles escaping at $T<15$, showing weak fluctuations in the energy is depicted in Fig. 12(b).

The scene is quite different in the case of noise intensity $\xi_{t}$. The fluctuations in the energy allow some particles to move in the scattering region with a wide range of average energies. In Fig. 13(a) the histogram shows a Gaussian distribution of the average energies. The distribution 
(a)

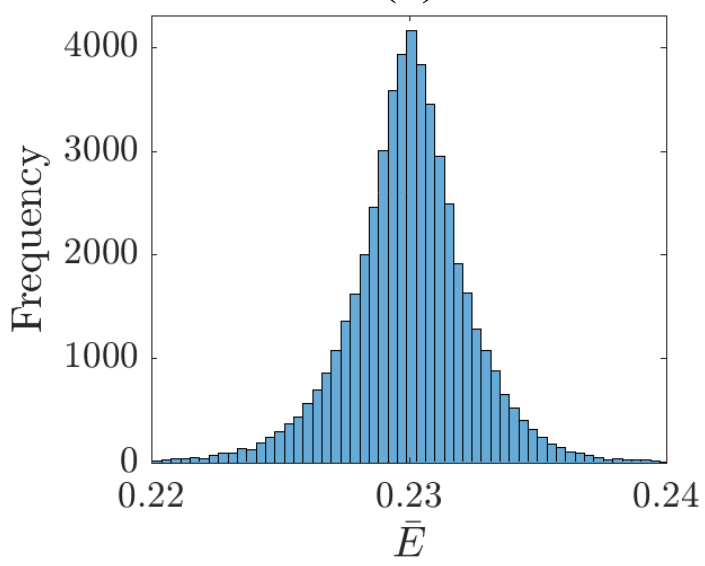

(b)

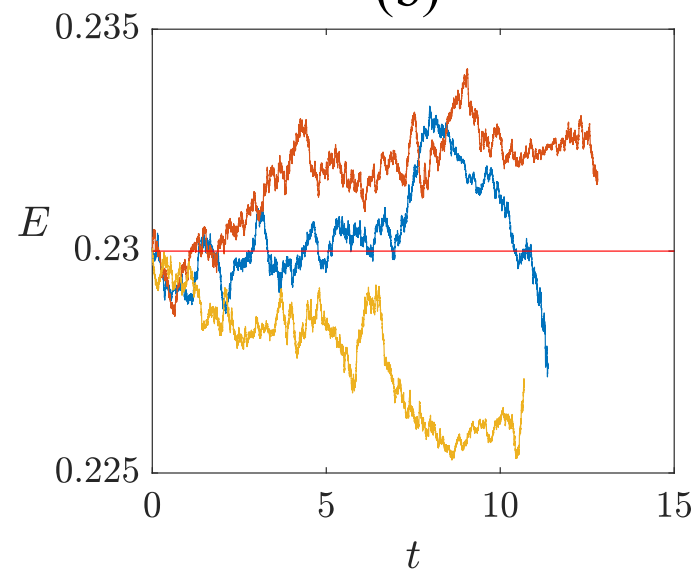

FIG. 12. (a) Histogram showing the distribution of the average energies of the particles in the system with $E=0.23$ and a weak noise of intensity $\xi=10^{-6}$. (b) Three usual particles for the same values of energy and noise, escaping in short times after weak fluctuations. The red line indicates the original value of the energy.

has a maximum at $E=E_{0}$, as in the case of weak noise, but the average energy ranges from $E=$ 0.1 to $E=0.3$. The Gaussian is incomplete in the right side due to the impossibility of remaining in the scattering region when having very high energies. This distribution of average energies means that some unusual trajectories can decrease their energy and remain in the scattering region during long transients. These particles are the ones that enhance the trapping. The lower the energy, the smaller the exit set and more probable is to spend long times in the potential. Moreover, as we have mentioned in the introduction, for energies $E<E_{e}=1 / 6$ the exits are closed and the particles cannot escape. In order to visualize this process, in Fig. 13 (b) the evolution of the average escape energy of one of these unusual trajectories is represented. This particle, that should escape in a time $T \approx 25$ in the deterministic system, decreases its energy, moving most of the time with $E<E_{e}$. After a very long transient, the fluctuations lead to an increasing in the energy and the particle escapes in $t \approx 1500$.

In the case of low noise intensities the trajectories have not the option of decrease noticeably the energy due the weak fluctuations. In the trapping the fluctuations are large enough to allow some particles to be trapped with energies $E<E_{e}$, or simply describe large transients with low energies. The bigger the energy, higher the increment $\Delta E$ necessary to reach low energy levels that allow the particle to remain in the scattering region. This is the reason that the value of $\xi_{t}$ 
(a)

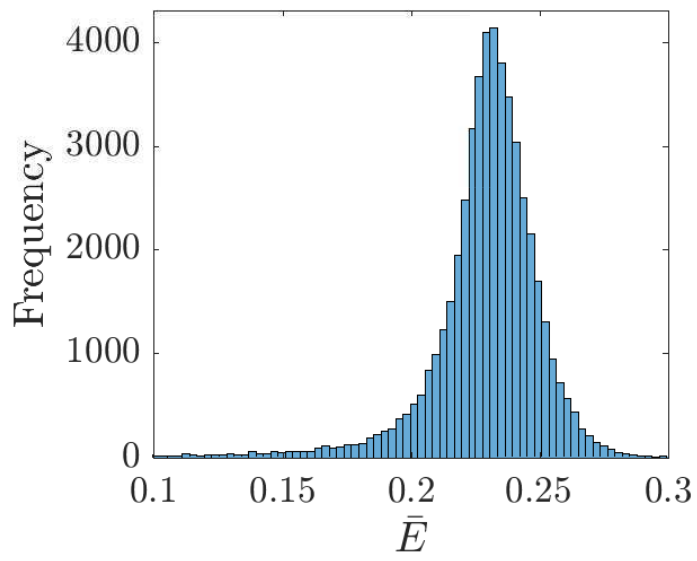

(b)

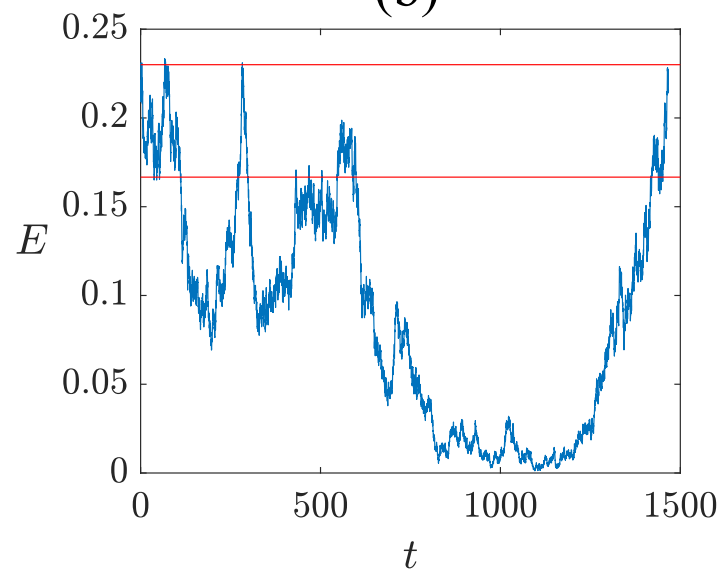

FIG. 13. (a) Histogram showing the distribution of the average energies of the particles in the system with $E=0.23$ and the noise intensity that enhances the trapping, $\xi_{t}=6.31 \times 10^{-5}$. (b) An unusual trajectory remaining in the scattering region during almost $t=1500$. The upper red line indicates the original energy, while the lower refers to the threshold, $E_{e}$, in which the isopotential curves are opened.

increases with increasing values of the energy.

At this point we could ask, why in the presence of high noise the fluctuations do not lead to an increasing of the average escape times, but the opposite? Under the influence of high intensity noise, the system can reach very low energy values. However, very violent fluctuations in the energy appear and the particles eventually escape in short times. This generates a decrease in the average escape time by increasing the energy to very high values where the escape time of the deterministic system is close to zero. To show this, we compare the intensity of the fluctuations for a strong noise $\xi=10^{-3}$ and for $\xi_{t}$ in Fig. 14. In a very short time $(t=17)$ the violent fluctuations of the strongly noisy system (red curve) reach energies close to 0.15 and over 0.35 , leading to an escape in $t<20$. In the case of considering $\xi_{t}$ (blue curve) the fluctuations allow the particle to avoid the escape by slowly reducing the energy. In fact, this trajectory is the same that we have shown in Fig. 13 (b), so even if we plot the evolution of the energy only until $t=20$, the trajectory remains in the scattering region until almost $t=1500$.

This mechanism related to the drop in energy explains the noise-enhanced trapping in the range of energies that we have considered $(E \in[0.18,0.25]$ ), which is represents both regimes in the scattering problem. The decrease in the energy opens different paths that participate in the trapping. First, the smaller the energy, the smaller the size of the exits, so the average escape time is 


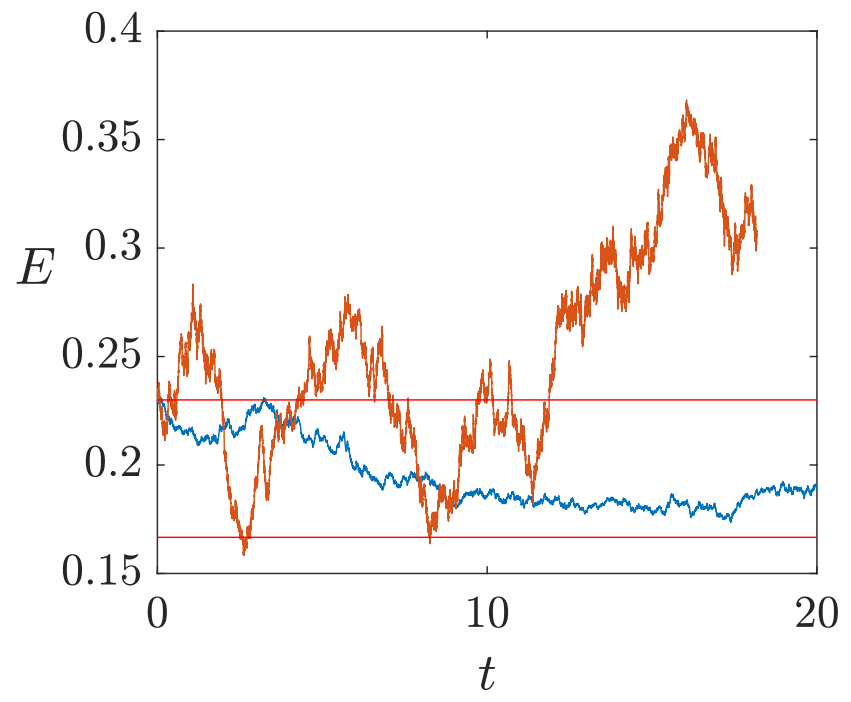

FIG. 14. Comparison between the fluctuations in the energy due to a strong noise $\xi=10^{-3}$ (red curve) and due to the noise that enhance the trapping $\xi_{t}=6.31 \times 10^{-5}$ (blue curve). The upper red line indicates the original energy $E=0.23$, while the lower refers to the threshold, $E_{e}$, in which the isopotential curves are opened.

decreased. On the other hand, for low energy values the KAM islands are bigger. Therefore, it is more probable that trajectories can jump inside one of them. Finally, in the Hénon-Heiles system and many other open Hamiltonian systems, the isopotential curves are closed under the threshold $E_{e}$, and hence the particles that reach such energy are trapped until a chain of positive fluctuations generates the escape. Since the main factors generating the trapping are more relevant for low energy levels, the more we increase the energy of the deterministic system, the greater the noise required to achieve the trapping. If we increase the energy more and more over $E=0.25$, we will need huge noise intensities to reach the trapping. These noise intensities could be physically meaningless. 


\section{CONCLUSIONS}

To summarize, our research reveals that a new mechanism that enhances the trapping occurs in continuous open Hamiltonian systems. To show this, we have provided both strong numerical evidence and theoretical arguments. The numerical simulations have been based on the average escape time of the particles and the probability distributions of the energy and the escape time. In the case of the mixed-phase-space regime, we have also shown the gradual reduction of the stickiness of the KAM islands for noise intensities weaker than the noise-enhanced trapping.

We have shown that this characteristic of chaotic scattering is related to the influence on the escape times of some unusual particles that decrease their energy due to the stochastic fluctuations, leading to a very long transient. We have provided numerical evidence by using the Hénon-Heiles Hamiltonian. However, we expect that this result can appear, with different values of the noise and different intensities of the trapping, in almost all continuous open Hamiltonian systems. In particular, the so called Barbanis potential [32], with applications to astrophysics [36, 37] and quantum mechanics [38], and other Hamiltonians used to model galactic movements [36, 39] are perfect candidates to exhibit this phenomenon.

We expect that this work could contribute to the general understanding of the effects of noise

in chaotic problems. We also hope that these results can have an application in many fields of science in which the noise models the effect of internal irregularities or the coupling of the system with the environment. Some examples are chaotic advection of fluids [40-42] and prey-predator competition models [43].

\section{ACKNOWLEDGMENTS}

This work has been supported by the Spanish State Research Agency (AEI) and the European Regional Development Fund (ERDF, EU) under Projects No. FIS2016-76883-P and No. PID2019105554GB-I00.

[1] Y.-C. Lai and T. Tél, Transient Chaos: Complex Dynamics on Finite-Time Scales (Springer, New York, NY, 2010). 
[2] T. Tél and M. Gruiz, Chaotic Dynamics: An Introduction Based on Classical Mechanics (Cambridge University Press, New York, NY, 2006).

[3] J. M. Seoane and M. A. F. Sanjuán, Rep. Prog. Phys 76, 016001 (2012).

[4] W. T. Lu, S. Sridhar, and M. Zworski, Phys. Rev. Lett. 91, 154101 (2003).

[5] E. Ott, Chaos in Dynamical Systems (Cambridge University Press, New York, NY, 1993).

[6] H.-J. Stöckmann, Quantum Chaos: An Introduction (Cambridge University Press, Cambridge, England, 1999).

[7] G. S. Ezra, H. Waalkens, and S. Wiggins, J. Chem. Phys. 130, 164118 (2009).

[8] S. Kawai, A. D. Bandrauk, C. Jaffé, T. Bartsch, J. Palacián, and T. Uzer, J. Chem. Phys. 126, 164306 (2007).

[9] T. Tél et al., Chaos 10, 89 (2000).

[10] I. Scheuring, T. Czárán, P. Szabó, G. Károlyi, and Z. Toroczkai, Orig. Life Evol. Biosph. 33, 319 (2003).

[11] A. B. Schelin et al., Phys. Rev. E 80, 016213 (2009).

[12] S. Bleher, C. Grebogi, and E. Ott, Physica D 46, 87 (1990).

[13] G. Contopoulos, H. E. Kandrup, and D. Kaufmann, Physica D 64, 310 (1993).

[14] H. E. Kandrup, C. Siopis, G. Contopoulos, and R. Dvorak, Chaos 9, 381 (1999).

[15] J. Aguirre, J. C. Vallejo, and M. A. F. Sanjuán, Phys. Rev. E 64, 066208 (2001).

[16] A. E. Motter and Y.-C. Lai, Phys. Rev. E 65, 015205(R) (2001).

[17] A. E. Motter, Y.-C. Lai, and C. Grebogi, Phys. Rev. E 68, 056307 (2003).

[18] J. M. Seoane, M. A. F. Sanjuán, and Y.-C. Lai, Phys. Rev. E 76, 016208 (2007).

[19] F. Blesa, J. M. Seoane, R. Barrio, and M. A. F. Sanjuán, Phys. Rev. E 89, 042909 (2014).

[20] A. R. Nieto, J. E. Alvarellos, J. M. Seoane, and M. A. F. Sanjuán, Phys. Rev. E 98, 062206 (2018).

[21] C. S. Rodrigues, A. P. S. de Moura, and C. Grebogi, Phys. Rev. E 82, 026211 (2010).

[22] R. M. da Silva, C. Manchein, and M. W. Beims, Phys. Rev. E 97, 022219 (2018).

[23] J. D. Bernal, J. M. Seoane, and M. A. F. Sanjuán, Phys. Rev. E 88, 032914 (2013).

[24] E. G. Altmann and A. Endler, Phys. Rev. Lett. 105, 244102 (2010).

[25] P. Mills, Commun. Nonlinear Sci. Numer. Simul. 11, 899 (2006).

[26] J. M. Seoane and M. A. F. Sanjuán, Phys. Lett. A 372, 110 (2008).

[27] C. Gan, S. Yang, and H. Lei, Phys. Rev. E 82, 066204 (2010).

[28] M. Hénon and C. Heiles, Astron. J. 69, 73 (1964). 
[29] R. Barrio, F. Blesa, and S. Serrano, Europhys. Lett. 82, 10003 (2008).

[30] J. C. Vallejo, J. Aguirre, and M. A. F. Sanjuán, Phys. Lett. A 311, 26 (2003).

[31] E. E. Zotos, Nonlinear Dyn. 79, 1665 (2015).

[32] R. Barrio, F. Blesa, and S. Serrano, New J. Phys. 11, 053004 (2009).

[33] A. R. Nieto, E. E. Zotos, J. M. Seoane, and M. A. F. Sanjuán, Nonlinear Dyn. 99, 3029 (2020).

[34] P. E. Kloeden, E. Platen, and H. Schurz, Numerical Solution of Stochastic Differential Equations (Springer, Berlin, 1994).

[35] G. Contopoulos, Astron. Astrophys. 231, 41 (1990).

[36] G. Contopoulos, Order and Chaos in Dynamical Astronomy (Springer, Berlin, 2002).

[37] J. F. Navarro, Sci. Rep. 9, 12174 (2019).

[38] D. Babyuk, R. E. Wyatt, and J. H. Frederick,J. Chem. Phys. 119, 6482 (2003).

[39] H. E. Kandrup, C. Siopis, G. Contopoulos, and R. Dvorak, Chaos 9, 381 (1999).

[40] J. P. Baltanás, A. Zaikin, F. Feudel, J. Kurths, and M. A. F. Sanjuán, Phys. Lett. A 297, 396 (2002).

[41] A. Daitche and T. Tél, Phys. Rev. Lett. 107, 244501 (2011).

[42] A. Daitche and T. Tél, New. J. Phys. 16, 073008 (2014).

[43] K. Das, K. S. Reddy, M. N. Srinivas, and N. H. Gazi, Appl. Math. Comput. 231, 117 (2014). 